\title{
Implications of Dynamic Pressure Transducer Mounting Variations on Measurements in Pyrotechnic Test Apparatus
}

\author{
Andreas Dibbern ${ }^{*}$ \\ NASA Kennedy Space Center, Florida 32899 \\ William Sipes ${ }^{\dagger}$ \\ Consultant, Canyon Country, California 91351 \\ Michael Hagopian ${ }^{\ddagger}$ \\ NASA Goddard Space Flight Center, Greenbelt, Maryland 20771 \\ Stephen H. McDougle ${ }^{\S}$ \\ NASA Test and Evaluation Contract, NASA Johnson Space Center White Sands Test Facility, \\ Las Cruces, New Mexico 88012 \\ Regor L. Saulsberry ${ }^{* *}$ \\ NASA Johnson Space Center White Sands Test Facility, Las Cruces, New Mexico 88012 \\ and \\ Jeffrey Crisafulli ${ }^{\dagger \dagger}$ \\ NASA Kennedy Space Center, Florida 32899
}

\begin{abstract}
Accurate dynamic pressure measurements are often difficult to make within small pyrotechnic devices, and transducer mounting difficulties can cause data anomalies that lead to erroneous conclusions. Delayed initial pressure response followed by data ringing has been observed when using miniaturized pressure transducer mounting adapters required to interface transducers to small test chambers. This delayed pressure response and ringing, combined with a high data acquisition rate, has complicated data analysis. This paper compares the output signal characteristics from different pressure transducer mounting options, where the passage distance from the transducer face to the pyrotechnic chamber is varied in length and diameter. By analyzing the data and understating the associated system dynamics, a more realistic understanding of the actual dynamic pressure variations is achieved. Three pressure transducer mounting configurations (elongated, standard, and face/flush mount) were simultaneously tested using NASA standard initiators in closed volume pressure bombs. This paper also presents results of these pressure transducer mounting configurations as a result of a larger NASA Engineering and Safety Center pyrovalve test project. Results from these tests indicate the improved performance of using face/flush mounted pressure transducers in this application. This type of mounting improved initial pressure measurement response time by approximately $19 \mu$ s over standard adapter mounting, eliminating most of the lag time; provided a near step-function type initial pressure increase; and greatly reduced data ringing in high data acquisition rate systems. The paper goes on to discuss other issues associated with the firing and instrumentation that are important for the tester to understand.
\end{abstract}

\footnotetext{
* Principal Engineer, NASA Engineering and Safety Center, NESC-2.

† Pyrotechnic Consultant, 18850 Laurel Crest Lane.

$\ddagger$ Chief Engineer for Applied Engineering, MS500.

$\S$ Senior Project Engineer, Laboratories Department, Bldg 201, P.O. Box 20, Associate Fellow.

${ }^{* * *}$ Project Manager, Laboratories Office/RF, P.O. Box 20, Senior Member.

${ }^{\dagger \dagger}$ Electrical Engineer for Ground Systems, NE-E3.
} 


\section{Nomenclature}

$2 P T=$ two-pressure-transducer (bomb tests)

$3 P T=$ three-pressure-transducer (bomb tests)

$N E S C=$ NASA Engineering and Safety Center

NSI $=$ NASA standard initiator

$P T=$ pressure transducer

$S T=$ special test identifier

$Y-P C A=$ Y-configured primer chamber/carrier assembly

\section{Introduction}

Accurate dynamic pressure measurements within small pyrotechnic devices can be challenging. Transducer mounting difficulties may lead to data anomalies and subsequent erroneous conclusions. Testing by NASA Engineering and Safety Center (NESC), performed during an investigation into booster ignition anomalies associated with a particular Y-configured primer chamber assembly (Y-PCA) ${ }^{\ddagger \ddagger}$ required the use of miniaturized transducer mounting adapters to interface pressure transducers to the small Y-PCA initiator cavities. ${ }^{1}$ During testing, observation of delayed initial cavity pressure response and excessive pressure spikes, followed by data ringing, required additional work to understand the data. Recognizing how and to what extent these adapters influence Y-PCA internal pressure data, coupled with the need for high transducer data acquisition rates, was crucial for analysis purposes and led to numerous tests using simulated test cavities (bombs). These tests demonstrated the effect on initial pressure rise, peak, and ringing in the test cavities. An analysis was performed on pressure output signal characteristics from three different pressure transducer mounting options, where the passage distance from transducer face to pyrotechnic cavity was varied in length and diameter. Mounting configurations tested (elongated, standard, and face/flush mounts) used NASA standard initiators (NSIs). The Y-PCA is an aluminum pyrotechnic device used to accommodate two initiators to direct or channel the individual output products of each initiator toward a booster charge. The booster charge, in turn, produces a sufficient amount of gas to activate a ram/valve assembly.

\section{Pressure Transducer Signatures in Y-PCA}

NESC Y-PCA testing was performed using Kistler ${ }^{\S \S} 603 \mathrm{~B} 1$ pressure transducers with Kistler 222P needle adapters combined with brass spacers to prevent adapter threads from breaking the internal PCA wall plane. The adapters provided a secure means to mount the transducer into the relatively thin PCA cavity sidewall without influencing cavity pressurization. This technique was based on previous successes with Lockheed Martin Space Systems Company. ${ }^{2}$ Adapter passages up to the cavity interface, including the drilled portion through the PCA parent material, were filled with Dow Corning ${ }^{\mathbb{B}^{* * *}} 33 \mathrm{M}$ grease to allow efficient pressure communication from the cavity to the transducer face (Fig. 1). Kistler 5010B charge amplifiers were used as the power source for the transducers.

Y-PCA pressure transducer data indicated high initial peak pressure spikes, followed immediately by "ringing" of data, dampening after approximately six cycles. This ringing was initially not understood, and efforts were taken to understand the phenomena because accurate pressure data were required for analysis. This investigation resulted in using a greased versus ungreased pressure transducer adapter tested within the same chamber initiator cavity. A dual NSI pressure chamber body is shown in Fig. 2.

Although initial peak pressures were approximately $6000 \mathrm{psi}$ less for the ungreased transducer (PT1), a curve fitted through both transducer traces (PT1 and PT2) indicated approximately the same initial peak pressure of 12,500 psig (Fig. 3).

\footnotetext{
${ }_{\ddagger}^{\ddagger}$ Due to confidentiality concerns the Y-PCA vendor in the References endnote has been designated as $x y z$. ${ }^{\$ \S \S \text { Kistler Instrument Corp., Amherst, NY, 888-KISTLER, http://www.kistler.com/ }}$

${ }^{* * *}$ Dow Corning ${ }^{\circledR}$ is a registered trademark of Dow Corning Corporation, Midland, Michigan.
} 


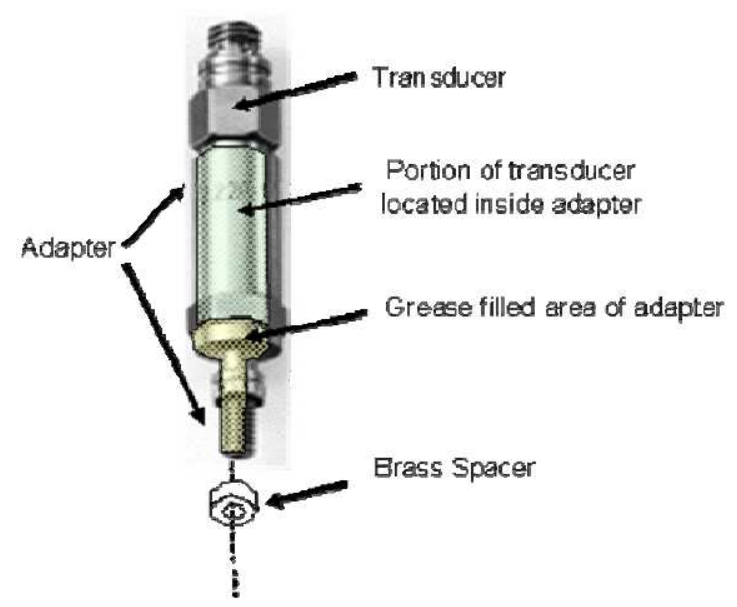

Figure 1. Relationship between the transducer and grease filled portion of adapter.

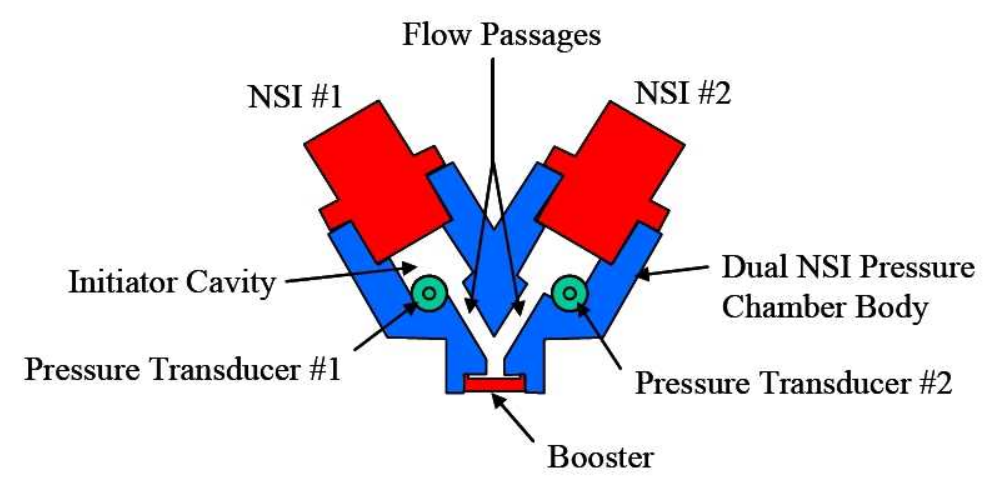

Figure 2. Dual NSI pressure chamber setup.

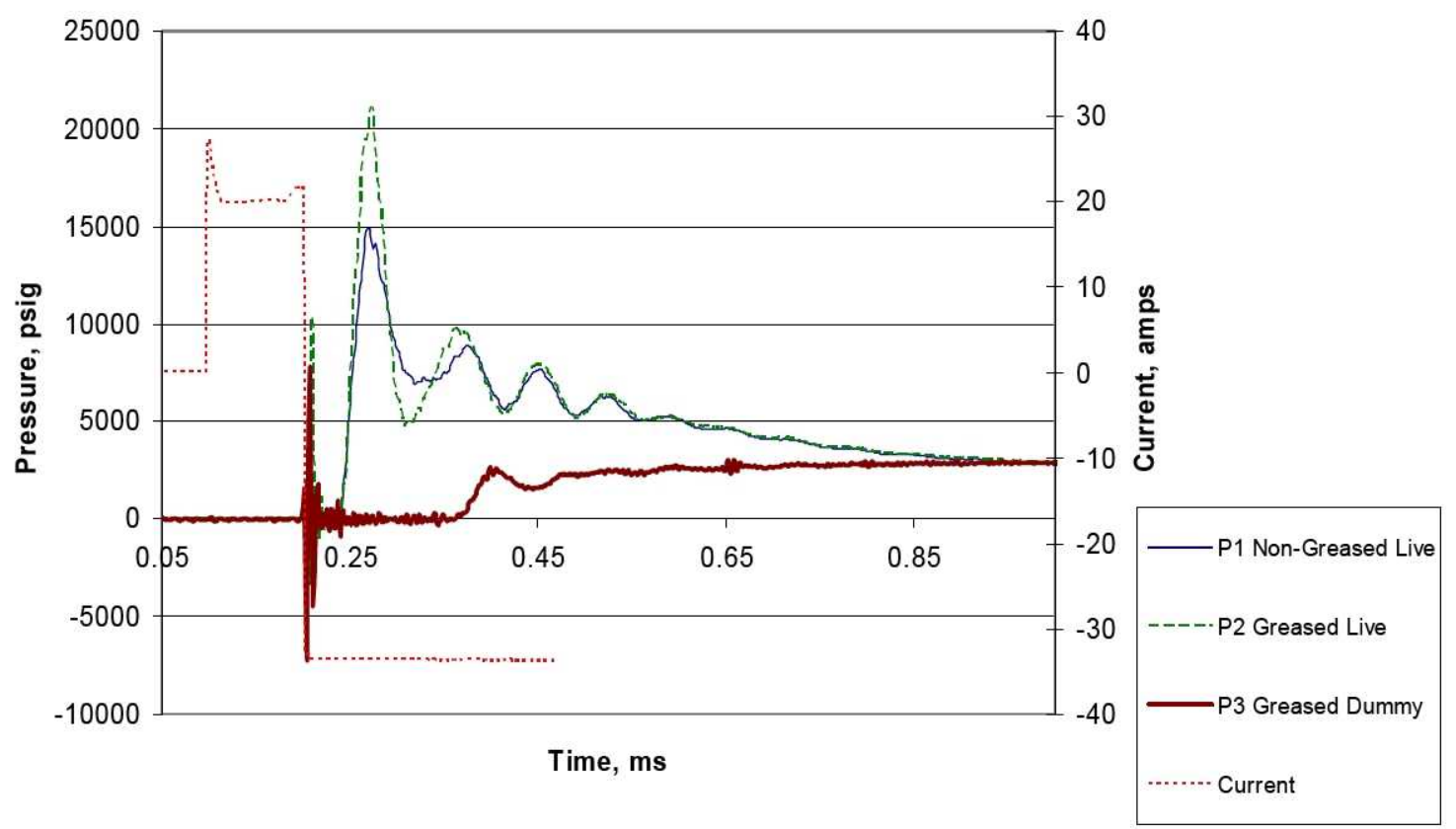

Figure 3. Plot of greased versus ungreased pressure transducer adapters (PT1 and PT2 in same cavity).

American Institute of Aeronautics and Astronautics 
Ringing was still noticed in the ungreased transducer at a frequency $(\sim 10$ to $13 \mathrm{KHz})$ similar to the greased transducer, indicating the ringing was not due to the addition of grease. It was later understood that, according to Kistler, the adapter had an inherent resonant frequency depending on the medium placed within the adapter (specifically the speed of sound in the medium). The resonant frequency observed during greased and ungreased transducer tests correlated well with what Kistler predicted with an air-filled (non-greased) adapter at $1500{ }^{\circ} \mathrm{F}$ $\left(816^{\circ} \mathrm{C}\right.$ ) (approximate temperature near transducer port during initiator firing). This indicated that the speed of sound was approximately the same for the grease and air (at higher temperature), suggesting the ringing observed during testing was due to the inherent resonance frequency of the adapter geometry.

To understand and possibly eliminate effects of the PCA or instrumentation internal geometry on the pressure transducer trace, additional tests using closed volume cavities (bombs) were developed.

\section{Two-Pressure-Transducer (2PT) Bomb Tests: Standard Mount}

Two different closed volume bombs (see Fig. 4) were initially produced to support two specific tests: one $0.9 \mathrm{cc}$ (test ST9) and one $0.5 \mathrm{cc}$ (test ST10). The primary emphasis of these tests was to understand what influence cavity volume change and shape change had on observed pressure trace ringing. These bombs were made out of tool steel to reduce or eliminate effects of melting and/or erosion due to initiator (NSI) heat input. (For a further discussion of the effects of the bomb material on test results, see AIAA 2008-4802, Material Properties Effects on Pyrotechnic Initiator Output. ${ }^{3}$ ) Each bomb had two pressure transducer ports, allowing additional investigation into the effect of mounting transducers 90 degrees apart. One Kistler 603B1 pressure transducer was mounted on the wall of the chamber using the same type of mounting adapter (Kistler 222P needle adapter) used during initial Y-PCA testing. The adapter mount flow passage, running through the bomb wall connecting the bomb cavity interface to the end of the adapter tip, had dimensions of $0.04 \mathrm{in}$. diameter and $0.04 \mathrm{in}$. length. The second transducer was mounted to the bottom of the bomb and slightly off center to avoid the initiator (NSI) end cap during firing, with the same mount configuration as the wall mounted configuration. Both adapter passages were filled with Dow Corning 33M grease. These tests are referred to as the two-pressure-transducer (2PT) bomb tests.

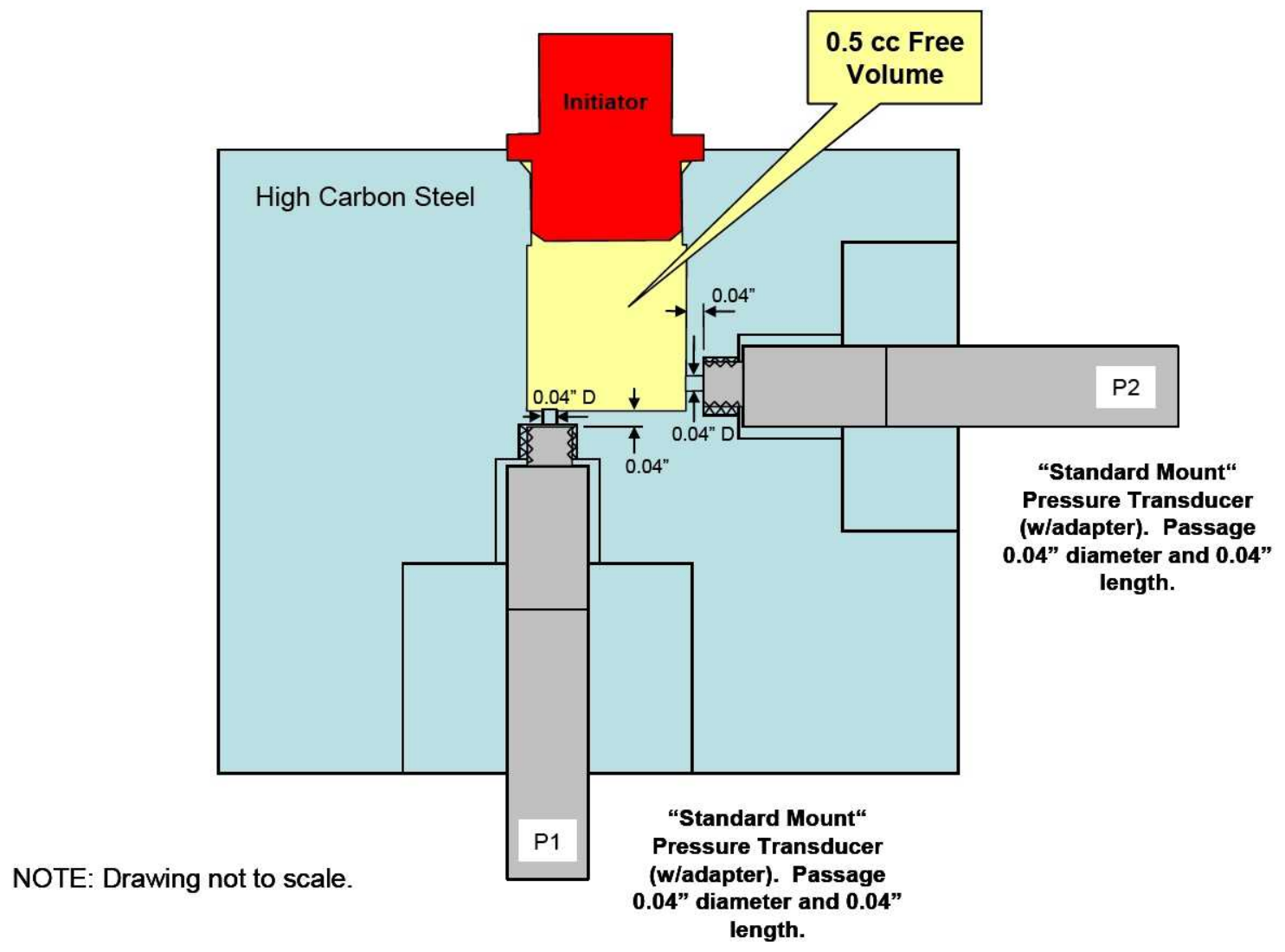

Figure 4. 0.5 cc $2 \mathrm{PT}$ bomb (the 0.9 cc $2 \mathrm{PT}$ bomb was similar in design). 
The 2PT bomb tests produced data traces highlighting similarities between the two standard mounting configurations. As the plot in Fig. 5 indicates, there is relatively little, if any, difference between the two pressure traces when $\mathrm{P} 2$ is slightly adjusted upward due to a pressure offset. Although indicated peak pressures range from 14,650 psig for P1 and 11,050 psig for P2, the peak pressure appears to be near 10,000 psig when an approximate curve-fit is applied to the traces. A more accurate pressure of $\sim 8000 \mathrm{psig}$ is observed outside of the influence of transducer ringing, $\sim 350 \mu \mathrm{s}$ after the initial pressure rise. Ringing took approximately three cycles to stabilize with a frequency of $\sim 12 \mathrm{KHz}$, which approached the PCA test frequency. Initial pressure increase or rise for the wall mounted transducer lagged $\sim 10 \mu \mathrm{s}$ on average for both tests. This suggests the wall and bottom mounted transducers had roughly the same pressure data values other than an initial pressure rise shift in time.

Increasing the cavity volume to $0.9 \mathrm{cc}$ had no effect on ringing characteristics other than lowering the pressure reading. The fact that the frequency and the cycles remained approximately the same, even though the length of the $0.9 \mathrm{cc}$ bomb was nearly twice the length of the $0.5 \mathrm{cc}$ bomb, eliminated a theory that the ringing pressure peaks were the result of a shock wave bouncing from one end of the bomb to the other.

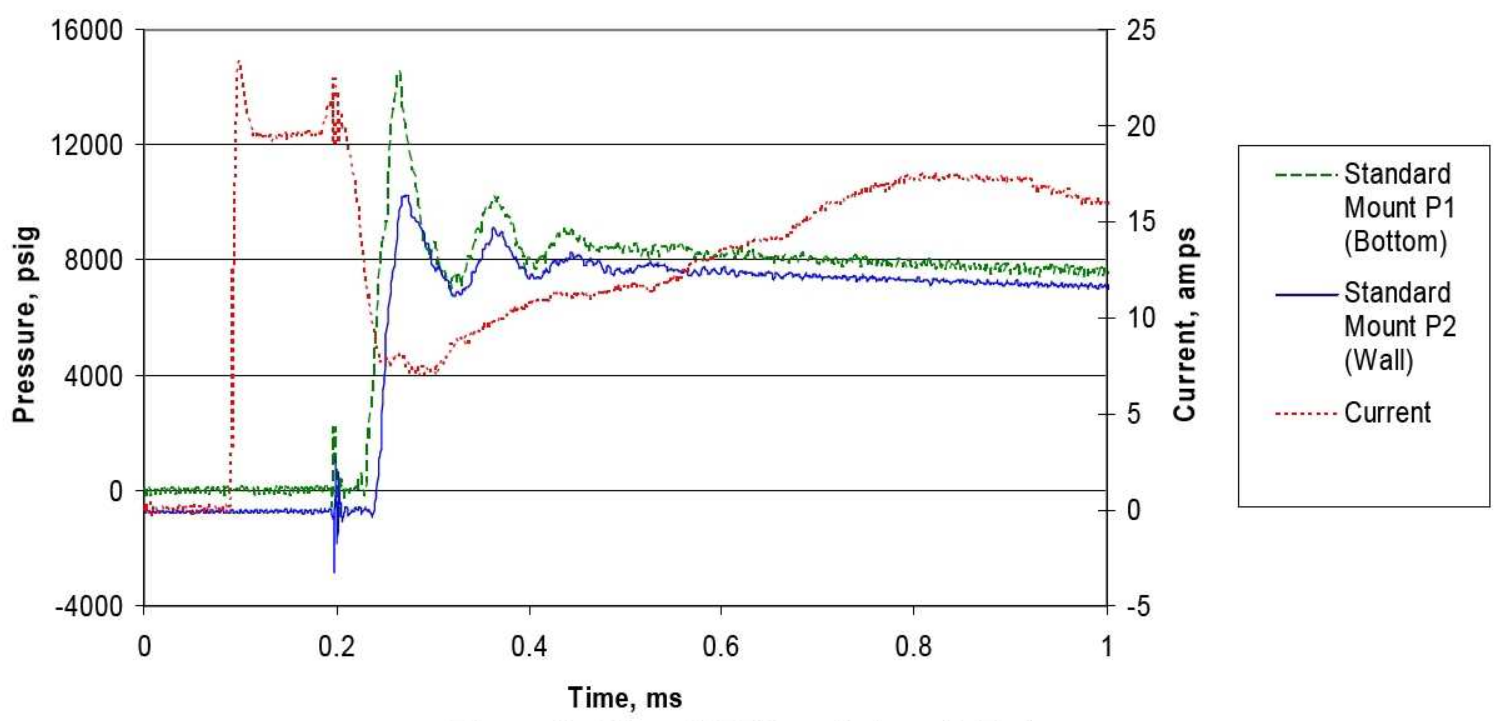

Figure 5. Plot of $2 \mathrm{PT}$ bomb data (0.5cc).

\section{Three-Pressure-Transducer (3PT) Bomb Tests: Standard, Elongated, and Face/Flush Mount}

In an effort to understand and reduce initial pressure rise delay, pressure spikes, and ringing, a third pressure bomb ( $0.5 \mathrm{cc}$ volume) was manufactured with a standard mount transducer located on the bottom utilizing the $222 \mathrm{P}$ adapter. The 222P adapter is an elongated mount wall transducer port that also uses the adapter as the standard mount, and an additional face/flush mount pressure transducer port that did not use an adapter (Fig. 6).

The elongated mount consisted of the drilled passageway connecting the bomb cavity to the adapter, increasing the length $0.25 \mathrm{in}$. beyond the standard mount configuration ( $0.29 \mathrm{in}$. total) while still maintaining the $0.04 \mathrm{in}$. standard adapter passageway diameter. The passageway for the face/flush mount setup (no adapter) had a diameter of $0.08 \mathrm{in}$., thereby greatly increasing the exposed face area of the pressure transducer. Although the face/flush mount was nearly flush with the internal bomb wall plane, a short passage length of $0.04 \mathrm{in}$. (same as standard mount) was designed into the bomb to accommodate a small amount of grease for transducer face protection.

Eight bomb tests (ST11 through ST16, STli, and ST18) were performed using the 3PT configuration. A representative 3PT bomb plot is shown in Fig. 7. This test configuration provided the most beneficial data on transducer mountings, as the three pressure transducers produced parallel results from one test. 


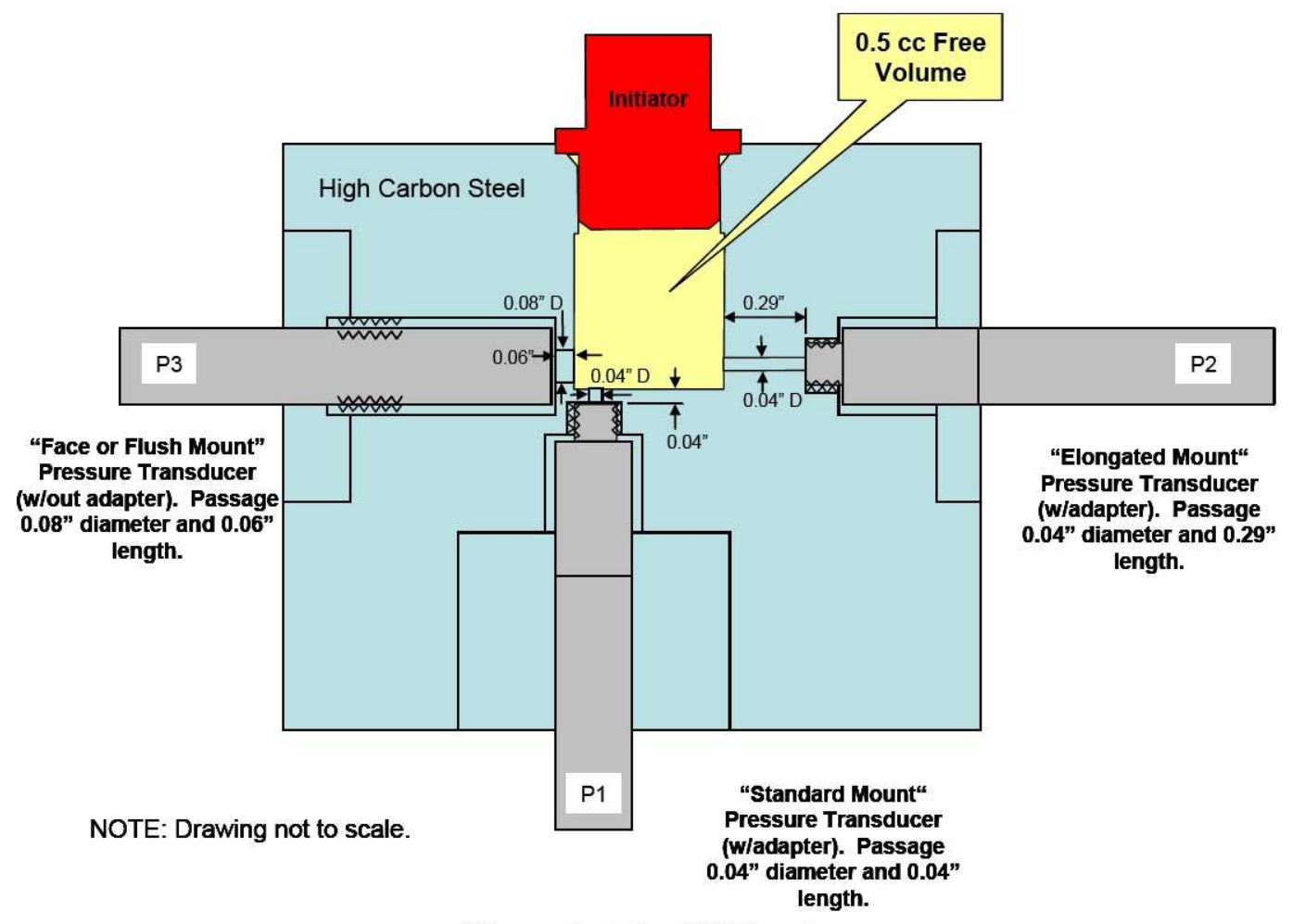

Figure 6. 0.5cc 3PT bomb.

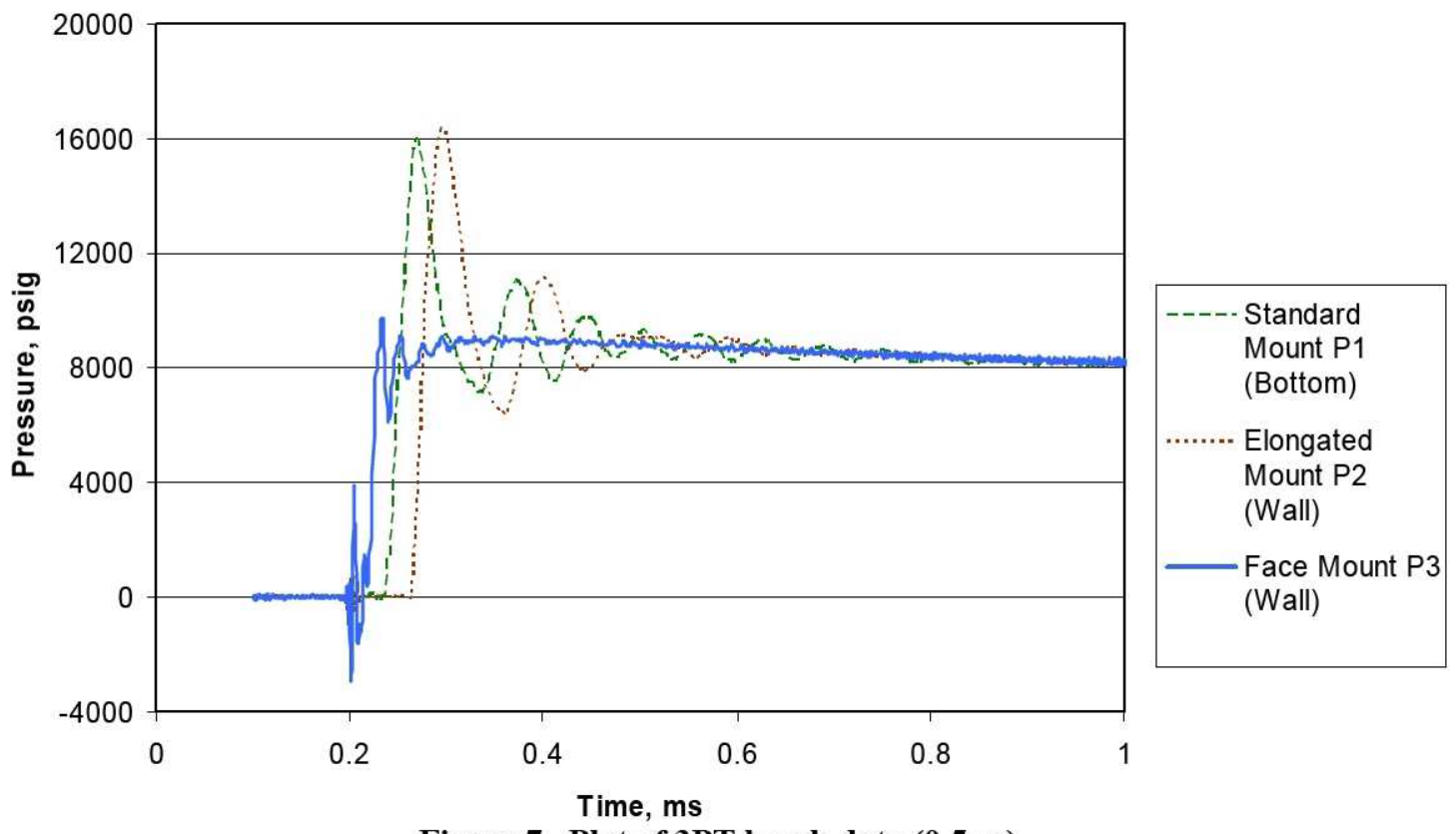

Figure 7. Plot of $3 P T$ bomb data $(0.5 \mathrm{cc})$.

The 3PT bomb test traces indicated that the initial pressure rise in the bomb chamber was always sensed by the face/flush mounted transducers first, followed by $18 \mu \mathrm{s}$ (on average) by the standard, and then $20 \mu \mathrm{s}$ (on average) by the elongated transducer mount. If the $\sim 10-\mu$ s lag time observed during the $2 \mathrm{PT}$ bomb tests between the standard wall and bottom mounted configurations is applied, the transducer timing sequence is still maintained. Another face/flush mounted transducer data characteristic indicates dramatically reduced high amplitude pressure spikes with initial pressure increases (within $100 \mu \mathrm{s}$ ) in an almost step-like fashion. Data for the 3PT bomb tests are provided in Table 1. 
Table 1. 3PT mounted bomb tests, 100 percent initiator (NSI).

\begin{tabular}{|c|c|c|c|c|c|c|c|c|c|c|c|}
\hline \multirow[b]{2}{*}{$\begin{array}{c}\text { Test } \\
\text { Number }\end{array}$} & \multicolumn{3}{|c|}{ "Face/Flush Mount" } & \multicolumn{4}{|c|}{ "Standard Mount" } & \multicolumn{4}{|c|}{ "Elongated Mount" } \\
\hline & $\begin{array}{c}\text { Peak } \\
\text { Pressure } \\
\text { (psig) }\end{array}$ & $\begin{array}{c}\text { Pressure at } \\
500 \mu s \\
\text { (psig) }\end{array}$ & $\begin{array}{c}\text { Time From } \\
\text { First to } \\
\text { Second } \\
\text { Oscillation } \\
\text { Peak } \\
\text { ( } \mu \text { s) }\end{array}$ & $\begin{array}{l}\text { Peak } \\
\text { Pressure } \\
\text { (psig) }\end{array}$ & $\begin{array}{c}\text { Pressure at } \\
500 \mu \mathrm{s} \\
\text { (psig) }\end{array}$ & $\begin{array}{l}\text { Time From } \\
\text { First to } \\
\text { second } \\
\text { Oscillation } \\
\text { Peak } \\
\text { (js) }\end{array}$ & \begin{tabular}{|c} 
Initial \\
Pressure \\
Increase with \\
Respect to \\
"Face/Flush" \\
Mount \\
Increase ( $\mu$ s)
\end{tabular} & $\begin{array}{c}\text { Peak } \\
\text { Pressure } \\
\text { (psig) }\end{array}$ & $\begin{array}{c}\text { Pressure at } \\
500 \mu \mathrm{s} \\
\text { (psig) }\end{array}$ & 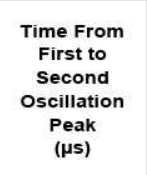 & $\begin{array}{c}\text { Initial } \\
\text { Pressure } \\
\text { Increase with } \\
\text { Respect to } \\
\text { "Face/Flush" } \\
\text { Mount } \\
\text { Increase (ps) }\end{array}$ \\
\hline 11 & 9,497 & 8,155 & 41.2 & 13,652 & 8,155 & 101.6 & 13 & 17,152 & 8,155 & 107.5 & 47 \\
\hline 12 & 9,765 & 8,716 & 20.3 & 16,010 & 8,716 & 103.2 & 19 & 16,333 & 8,716 & 100.7 & 47 \\
\hline 13 & 9,272 & 9,697 & 29.1 & 15,087 & 9,770 & 92.5 & 19 & Clipped & 9,255 & 98.9 & 41 \\
\hline 14 & 10,078 & 10,093 & 8.2 & 13,916 & 9,741 & 106.6 & 19 & Clipped & 9,846 & NA & 33 \\
\hline 15 & 8,657 & 9,667 & 8.8 & 15,103 & 9,199 & 105.4 & 19 & Clipped & 9,416 & NA & 33 \\
\hline 16 & 11,630 & 8,936 & 9.0 & 16,260 & 8,643 & 104.4 & 21 & Clipped & 8,639 & NA & 26 \\
\hline 18 & 10,655 & 9,563 & 6.6 & 14,270 & 9,375 & 58.5 & 12 & 12,327 & 8,902 & 68.2 & 27 \\
\hline $1 \mathrm{i}$ & 9,955 & 9,343 & 9.0 & 15,589 & 9,107 & 100.5 & 18 & 15,562 & 8,757 & 101.1 & 49 \\
\hline $\begin{array}{c}\text { Summary } \\
\text { Average }\end{array}$ & 9,939 & 9,271 & $17(59 \mathrm{KHz})$ & 14,986 & 9,088 & $97(10 \mathrm{KHz})$ & 18 & 15,344 & 8,961 & $95(10 \mathrm{KHz})$ & 38 \\
\hline
\end{tabular}

To compare the data between the three mounts without the effect of adapter-induced transducer ringing, a point $500 \mu \mathrm{s}$ after initial pressure rise was chosen. These data indicated good correlation, as all three pressure transducers were within 3.5 percent of one another. At this point, the average face/flush mounted peak pressure value was also quite near the initial indicated peak value (within 7 percent) for the eight NSI tests, while standard and elongated mount peak value relationships were much greater (65 and 71 percent, respectively). The initial pressure peak (plateau) produced by the face/flush mount was reasonably closer in value to the approximate curve-fit peak pressure value of the standard and elongated mounts than their respective indicated peak values. This suggests data from the face/flush mount yield a better representation of initial cavity pressure.

Oscillations on the face/flush mounted transducer traces indicated a reduced number of oscillations at much higher frequency (40 to $60 \mathrm{KHz}$ ) when compared to the other mounting configurations, and were almost completely damped within the first $100 \mu$ s after initial pressure rise and were of minimal amplitude. Average time between the oscillation peaks was shortest for the face/flush mount $(0.017 \mathrm{~ms}, 60 \mathrm{KHz})$, followed by the standard and elongated mount of approximate equal frequency $(0.095 \mathrm{~ms}, 11 \mathrm{KHz})$.

\section{Conclusion}

The effects of different pressure transducer mountings within the same bomb produced noticeable changes on pressure data traces, with a face/flush mounted configuration indicating the fastest response and most accurate data. Although data produced by pressure transducers using the miniature adapter mounts have accuracy limitations immediately after sensing pressure (due to ringing) and should not be considered the actual working pressure, the data provide a means of determining internal timing events, which is helpful in studying pressure changes in small pyrotechnic cavities.

\section{References}

\footnotetext{
${ }^{1}$ M. Hagopian and A. Dibbern, "Vendor xyz Y-PCA Booster Anomaly Investigation," NASA Engineering and Safety Center Report No. RP-08-111, NASA Technical Memorandum No. TM-2008-215548, 2008 (unpublished).

${ }^{2}$ T. Raynor et al. Failure Investigation Report, Pyrovalve Primer Chamber Assembly (PCA), PSPM-0343-U-06, Lockheed Martin Space Systems Company, Littleton, Colorado, August 10, 2006.

${ }^{3}$ T. J. Hinkel and F. Salazar, "Material Properties Effects on Pyrotechnic Initiator Output," Proceedings of the $44^{\text {th }}$ AIAA/ASME/SAE/ASEE Joint Propulsion Conference \& Exhibit, AIAA 2008-4802, American Institute of Aeronautics and Astronautics, Washington, DC, July 2008.
} 